\title{
OPTICAL PROPERTIES \\ OF A TWO-DIMENSIONAL ARRAY OF METALLIC SPHERES ON A SUBSTRATE
}

\author{
A. Modinos \\ Department of Physics, Zografou Campus \\ National Technical University of Athens \\ 15773 Athens, Greece \\ AND N. STEFANOU \\ Solid State Section, University of Athens \\ Panepistimioupolis, New Buildings \\ 15771 Athens, Greece
}

(Received May 21, 1991; in revised form June 5, 1991)

We describe a method for the calculation of the optical properties of a two-dimensional array of non-overlapping metallic particles (approximated by spheres) adsorbed on a dielectric slab. The interest in such systems arises to a large degree from their possible use as coatings, e.g. for solar energy absorbers and similar technological purposes. The formalism is an extension of the methods which have been developed in relation to electron scattering by two-dimensional atomic layers and takes fully into account multiple scattering of light between the particles of the overlayer and between the overlayer and the substrate. Scattering of light by multilayers or by an infinite crystal of non-overlapping spheres can be dealt with by a straightforward extension of the theory as in the theory of low-energy electron diffraction. Our calculations show that the usual approximation of replacement of the metallic particles by effective dipoles fails when the size of the particles or the concentration of particles increases beyond a limit and that $\ell$-pole contribution in interparticle scattering beyond the dipolar $(\ell=1)$ one introduces new structure in the absorbance versus frequency curve. The reflection and absorption of light as a function of frequency is obtained numerically for selected examples. We consider in particular the variation of these quantities with concentration coverage. We examine also the effect of disorder.

PACS numbers: 78.65.-s 


\section{Introduction}

In the last few years it has been discovered that films consisting of small metallic particles embedded in a dielectric host material have optical properties that might be useful in a variety of technological applications, e.g. as coatings for solar energy absorbers [1].

Calculations relating to the optical properties of inhomogeneous systems consisting of non-overlapping metallic spheres of $\approx 100 \AA$ radius in a dielectric host have been published by a number of authors (see $[2,3]$ and references therein). In most of these calculations the metallic spheres are approximated by dipoles, with a Drude-type polarisability, leading to an effective dielectric function for the composite medium via the Clausius-Mossotti equation or variations of it. These calculations are not valid when the size of the particles and/or the interparticle distance is of the order of the incident light wavelength or when the fractional volume occupied by the spheres exceeds a half or so.

Lamb et al. [4] were the first to attempt a treatment of the propagation of light in the composite medium which solves Maxwell's equations and therefore takes into account $\ell$-pole tcrms above the dipole $(\ell=1)$ one in the evaluation of the interparticle scattering of light. Their analysis employs a modified version of the Korringa-Kohn-Rostoker (KKR) procedure used in the calculation of electron-energy-bands in solids. It yields an effective propagation wave vector in an infinite crystal and as such is related to a transmission experiment in the forward direction but it does not describe a real experiment because it does not provide for a proper matching of the incident electromagnetic wave to the reflected and transmitted into the crystal waves at the surface.

A method which makes this possible has been formulated by us in Refs. $[2,3]$ and its applicability demonstrated by application to specific examples [3, 5]. The basis of it is the calculation of the reflection and transmission matrix elements, for light incident at a given angle, of a two-dimensional array (periodic to begin with) of spheres by a mathematical procedure which can be formulated as an extension of the metliods which have been developed in relation to electron scattering by two-dimensional atomic layers $[6,7]$. In many applications the system under consideration consists of a plane of spheres on a dielectric slab with a given dielectric constant. The reflection; absorption and transmission of light by such a system is obtained in a straightforward manner from the matrix elements that describe the scattering of light by the plane of spheres and those of the substrate slab [3]. The examples given in Sec. 3 are examples of such systems.

We note, however, that knowing the matrix elements for a layer (a plane) of spheres, we can obtain those of two layers, four layers etc., using a doubling layer scheme as in electron scattering by crystals $[6,7]$. Alternatively, we can obtain the complex band structure for electromagnetic waves and through it the reflection of light by a "crystal" of metallic or dielectric spheres. This relates to the photonic gap problem which has attracted considerable attention recently [8]. We shall not deal with this problem here. 


\section{Scattering by a plane of spheres}

We consider a two-dimensional array of metallic spheres centred on the sites of a periodic lattice in the $x y$-plane, embedded in a dielectric host material. We assume that a plane electromagnetic wave whose electric field component is given by

$$
\boldsymbol{E}_{0}(\boldsymbol{r}) \exp (-\mathrm{i} \omega t)=\boldsymbol{E}_{0}(k) \exp (\mathrm{i} k \cdot \boldsymbol{r}-\mathrm{i} \omega t)
$$

(the expression for the magnetic field $H=(-\mathrm{i} / \omega \mu) \nabla \times \boldsymbol{E}$ associated with a given electric field will not be written down), is incident upon this array of spheres. The electric field component of the scattered wave is given by (we omit the time factor $\exp (-\mathrm{i} \omega t))$

$$
\begin{array}{r}
\boldsymbol{E}_{\mathrm{s}}(\boldsymbol{r})=\sum_{\boldsymbol{R}_{n}} \sum_{\ell m} \exp \left(\mathrm{i} k_{\|} \cdot \boldsymbol{n}_{n}\right)\left\{a_{\ell m}^{\mathrm{s} I} L_{n} h_{\ell}^{+}\left(k r_{n}\right) Y_{\ell m}\left(\hat{r}_{n}\right)\right. \\
\left.+\frac{\mathrm{i}}{k} a_{\ell m}^{\mathrm{sE}} \nabla \times\left[L_{n} h_{\ell}^{+}\left(k r_{n}\right) Y_{\ell m}\left(\hat{r}_{n}\right)\right]\right\} .
\end{array}
$$

We note that the magnitude of the wave vector $k=\sqrt{\mu \varepsilon} \omega$ where $\varepsilon$ and $\mu$ denote the dielectric constant and magnetic permeability of the host material; $k_{\|}$is the component of $k$ in the $x y$-plane; $R_{n}$ denotes the centre of the $n$-th sphere, $r_{n} \equiv$ $r-R_{n}$ and $\hat{r}_{n}$ stands for the angular variable of $r_{n} \cdot L_{n} \equiv-\mathrm{i} r_{n} \times \nabla_{n}$ is the operator for the angular momentum with respect to $\boldsymbol{R}_{n}$ and depends only on $\hat{r}_{n}$, so that

$$
L_{n} h_{\ell}^{+}\left(k r_{n}\right) Y_{\ell m}\left(\hat{r}_{n}\right)=h_{\ell}^{+}\left(k r_{n}\right) L_{n} Y_{\ell m}\left(\hat{r}_{n}\right)
$$

which makes the mathematical analysis a lot easier. As usual $Y_{\ell m}$ denotes a spherical harmonic and $h_{\ell}^{+}$a spherical Hankel function. The coefficients $a_{\ell m}^{\mathrm{sH} H(E)}$ which give the amplitude of the $\ell m$-component of the scattered wave from the (zero)th sphere (the sphere centred on the origin), are determined by the $\ell m$-component of the electromagnetic wave incident on this sphere, by applying standard boundary conditions on the surface of the sphere. We have

"Electric field incident on the (zero)th sphere"=

$$
=E_{0}(k) \exp (\mathrm{i} k \cdot r)+E_{\mathrm{s}}^{\prime}(r)
$$

with a corresponding formula for the incident magnetic field. The second term on the right hand side of Eq. (4) is due to the waves scattered from all the spheres in the plane except the one at the origin and it is given by Eq. (2) with the term corresponding to $R_{n}=0$ excluded. Since $E_{\mathrm{s}}^{\prime}(r)$ is a function of the $a_{\ell m}^{\mathrm{s} H(E)}$ coefficients one obtains a self-consistent system of equations which one must solve to obtain these coefficients. The central problem in the implementation of this procedure is to expand the field given by Eq. (4) into spherical waves about the origin. The expansion of the first term (incident plane wave) is straightforward. The second term can be written as a sum of spherical waves about the origin as follows:

$$
\boldsymbol{E}_{\mathrm{s}}^{\prime}(\boldsymbol{r})=\sum_{\ell m}\left[a_{\ell m}^{\prime H} L j_{\ell}(k r) Y_{\ell m}^{\prime}(\hat{r})+\frac{\mathrm{i}}{k} a_{\ell m}^{\prime E} \nabla \times L j_{\ell}(k r) Y_{\ell m}(\hat{r})\right]
$$


where $j_{\ell}(k r)$ denotes, as usual, a spherical Bessel function. It can be shown that $[2,3]$

$$
a_{\ell^{\prime} m^{\prime}}^{\prime E}=\sum_{\ell m}\left(\Omega_{\ell^{\prime} m^{\prime} ; \ell m}^{(1)} a_{\ell m}^{s E}-\Omega_{\ell^{\prime} m^{\prime} ; \ell m}^{(2)} a_{\ell m}^{s I}\right) .
$$

A similar formula gives $a_{\ell^{\prime} m^{\prime}}^{\prime H}$ in terms of $a_{\ell m}^{s H(E)}$. The matrix elements of $\Omega^{(1)}$ and $\Omega^{(2)}$ depend of course on the scattering properties of the individual sphere, the geometry of the plane and on the frequency and wave vector of the incident radiation. The $\Omega$-matrices have certain symmetry properties which facilitate their calculation [3]. Furthermore, it turns out that these matrices involve quantities which are either identical or similar to those met in the treatment of electron scattering by a layer of muffin-tin atoms which allows one to use, to some degree at least, existing programs [6] for the computation of these quantities.

Using standard formulae one can rewrite the scattered wave of Eq. (2) as a sum of plane waves and thus obtain the scattering matrix elements in this representation.

In many experiments the array of metallic spheres sits on a substrate which is a dielectric plate of thickness $d$ and permittivity $\varepsilon_{\mathbf{s}}$. The situation is described schematically in Fig. 1. The incident wave of frequency $\omega$ has a wave vector with a component parallel to the plane of the spheres denoted by $k_{\|}+g_{0}$, where $k_{\|}$ lies within the surface Brillouin zone of the corresponding two-dimensional lattice, and $g_{0}$ is a reciprocal lattice vector. The amplitude of the wave is denoted by $\left[E_{0}\right]_{g_{0}} i$ where $i$ indicates a polarisation direction. Because of the two-dimensional periodicity of the structure the reflected and transmitted light consists of a number of beams (plane waves) corresponding to different reciprocal vectors $g$ of the given lattice. $\left[E_{\mathrm{rr}}\right]_{g_{i}}$ is the amplitude of a reflected beam with a wave vector parallel to the surface equal to $k_{\|}+g$. The same applies to the transmitted beam. The matrix elements which relate an incident plane wave to a transmitted or reflected one by the array of spheres are denoted by $M_{g_{i} ; g^{\prime} i^{\prime}}^{+}$etc., which are functions of the $a_{\ell m}^{\mathrm{s} H(E)}$ coefficients of Eq. (2) and the geometry of the layer. The (diagonal) matrices $\mathcal{R}$ and $\mathcal{T}$ which describe reflection and transmission, respectively, by the dielectric plate can be combined with the $\mathcal{M}$ matrices to give the reflecticity, transmittance and absorbance of the entire system: spheres on the substrate. The detailed formulae are given in Ref. [3]. We note that when the frequency of the incident wave is sufficiently low, so that $k \ll|g|$ for $g \neq 0$, only the $g=0$ term will contribute to the scattered wave.

\section{Examples}

In Fig. 2 we show the absorbance, calculated as a function of frequency, of $p$-polarized light by a square array of silver spheres for three different values of the sphere radius $S$ with the lattice kept constant (lattice constant: $a=350 \AA$ ). In Fig. 3 the absorbance is given for four different values of $a$, for given $S=80 \AA$. We assumed that the optical responce of the individual mettalic sphere is adequately described by the Drude dielectric function:

$$
\varepsilon_{M}(\omega)=1-\frac{\omega_{p}^{2}}{\omega(\omega+\mathrm{i} / \tau)}
$$




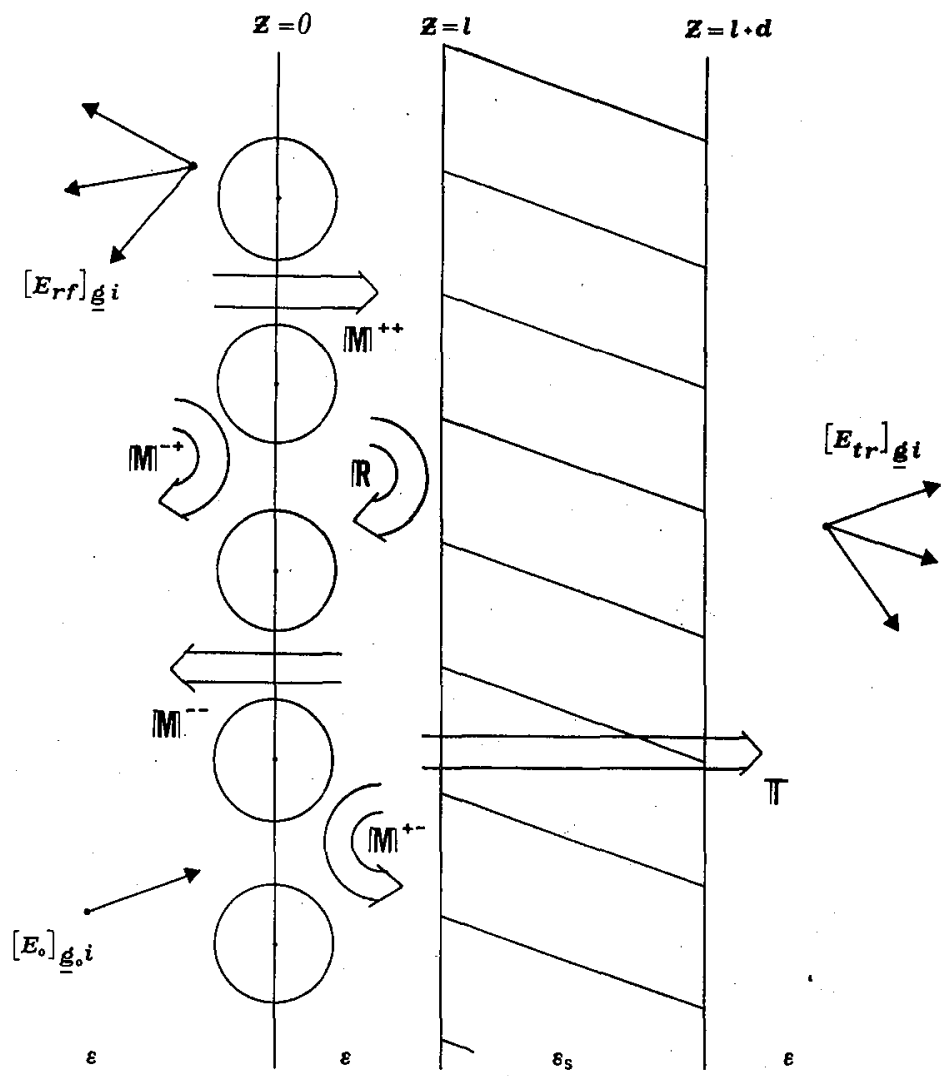

Fig. 1. Scattering of a plane wave from a periodic monolayer of spherical particles on a substrate.

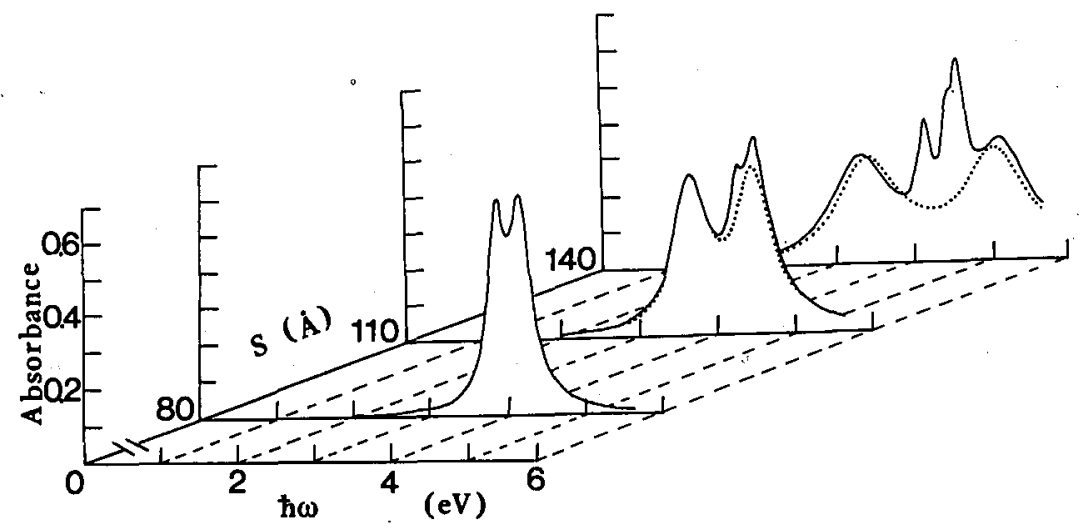

Fig. 2. Absorbance of $p$-polarised light of angular frequency $\omega$ incident at an angle $\theta=\pi / 4$ on a square array of silver spheres with the lattice constant $a=350 \AA$ for various sphere radii $S$. $\left(\longleftarrow \ell_{\max }=4 ; \cdots \cdots \ell_{\max }=1\right)$. 


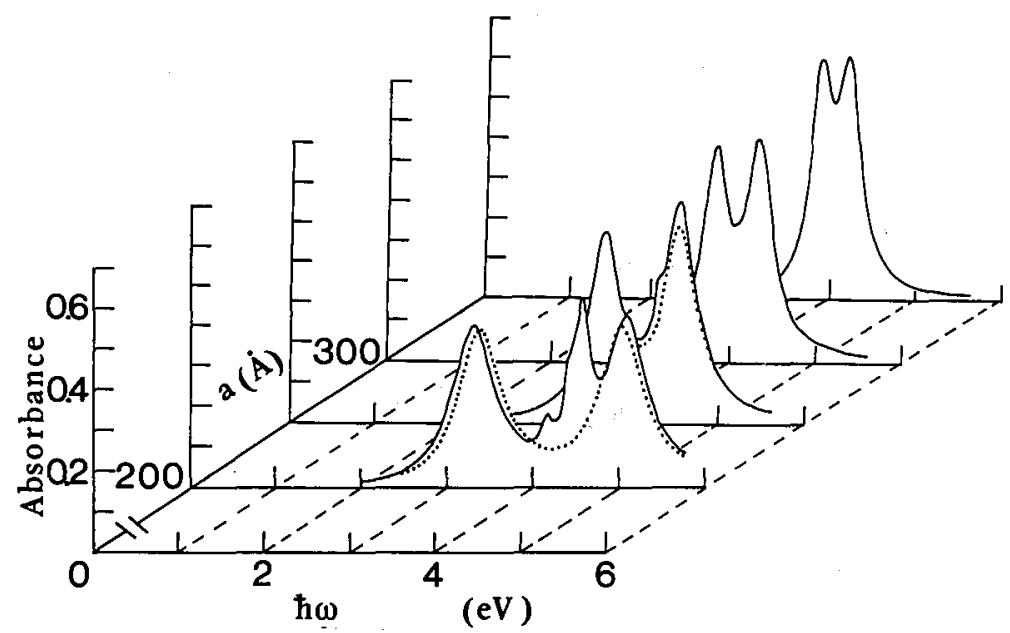

Fig. 3. Absorbance of $p$-polarised light of angular frequency $\omega$ incident at an angle $\theta=\pi / 4$ on a square array of silver spheres of radius $S=80 \AA$ for various lattice constants $a .\left(-\ell_{\max }=4 ; \cdots \cdots \ell_{\max }=1\right)$.

Following Persson and Liebsch [9] we have taken $\hbar \omega_{p}=6.93 \mathrm{eV}$ and $\hbar \tau^{-1}=$ $0.158 \mathrm{eV}$ which, it is assumed, are appropriate for silver particles. In both cases the dotted curve is calculated in the dipole approximation, i.e. only the dipole $(\ell=1)$ term is kept in the expansion of Eq. (2). The low energy peak corresponds to a parallel mode resonance, i.e. charge oscillates parallel to the plane of the spheres, induced by the component of the electric field parallel to this plane. The high energy peak corresponds to a normal mode resonance, i.e. charge oscillates normal to the plane of the spheres, induced by the component of the electric field normal to this plane. We see that as the sphere radius increases (Fig. 2) or as the lattice constant decreases (Fig. 3), i.e. as the coverage increases, the parallel mode resonance is shifted towards lower frequencies and the normal mode resonance to higher frequencies which is in agreement with available experimental data [10]. Inclusion of $\ell$-pole terms above the dipolar leads to additional structure as $S / a$ increases, as shown by the solid lines in Figs. 2 and 3 . Convergence is obtained for $\ell_{\max }=4$, i.e. by including in the expansion of Eq. (2) all terms up to and including those with $\ell_{\max }=4$. The $\ell$-pole $(\ell>1)$ contribution to the absorbance of the array of spheres comes through interparticle scattering of light which determines the total wave field incident on a given sphere. This additional structure has not been observed experimentally so far. We suspect that this structure may be very sensitive to the shape of the metal particles and that deviation from the spherical shape may destroy it (see also Ref. [5]).

We have assumed an array with a two-dimensional periodicity so far. There are experiments where this is so, at least to a good approximation. In Ref. [3] we presented an analysis of relevant experimental data by Craighead and Niklasson 
[11] on the scattering of light by a square array of nearly spherical gold particles on a sapphire substrate and found reasonably good agreement between theory and experiment. In many cases, however, the metallic particles are not arranged periodically on the substrate and one has to take into account the effect of disorder on the optical properties of the system. The simplest way to introduce disorder into the calculation is to assume that the metal spheres occupy randomly a fraction, denoted by $c$, of the sites of a regular two-dimensional lattice and that the remaining sites are empty. In the average $T$-matrix approximation (ATA) the partly empty lattice is replaced by a fully occupied one and the $T$-matrix which describes the scattering of light by a single sphere (for a proper definition of the $T$-matrix see Ref. [3]) is replaced by an average matrix given by $c T$. One obtains a measure of the effect of disorder by comparing the absorbance of light by the disordered partly empty lattice with a lattice constant $a$, with that calculated for a fully occupied lattice with a lattice constant $a^{\prime}=a / \sqrt{c}$ so that the coverage is the same in both cases. This is done in Fig. 4 for $p$-polarised light. We see that disorder pushes

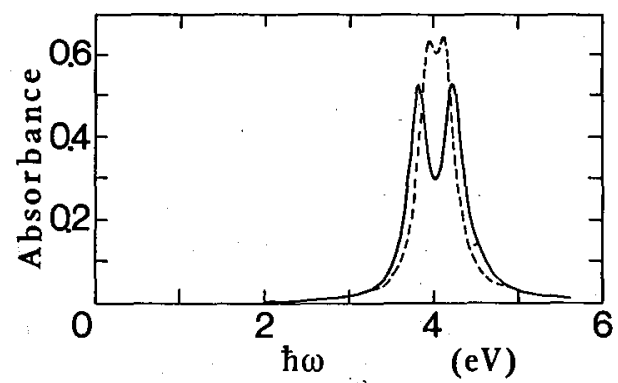

Fig. 4. Absorbance of $p$-polarised light of angular frequency $\omega$ incident at an angle $\theta=\pi / 4$ on: (i) a disordered array of silver spheres of radius $S=80 \AA$ occupying randomly $25 \%$ of the sites of a square lattice with the lattice constant $a=200 \AA$ (full line) and (ii) an ordered square array of silver spheres of radius $S=80 \AA$ with the lattice constant $a=400 \AA$ (dashed line).

the normal mode peak (higher in energy) further up to higher frequencies and the parallel mode peak (lower in energy) further down to lower frequencies. The effect of disorder on the absorbance curve of a two-dimensional array of spheres has also been considered by Persson and Liebsch [9] in the dipole approximation using an approximate version of the coherent potential approximation (CPA) method. It turns out that the shifts predicted by the ATA calculation are in the same direction but smaller, especially in the case of the parallel mode, than those of the CPA calculation. A reliable comparison with experimental data can not be made at this stage because of other factors which enter into the determination of the experimental spectra.

We have also examined the effect of the substrate (a dielectric slab) on the absorbance of the system: substrate plus adsorbed spheres. We considered a square lattice with a fraction $c=0.75$ of its sites randomly occupied on a substrate of 
polyvinyl alcohol (PVA) which has a dielectric constant $\varepsilon_{\mathrm{s}}=2.25$. The result of the calculation for light incident normally on the system is shown in Fig. 5 . The

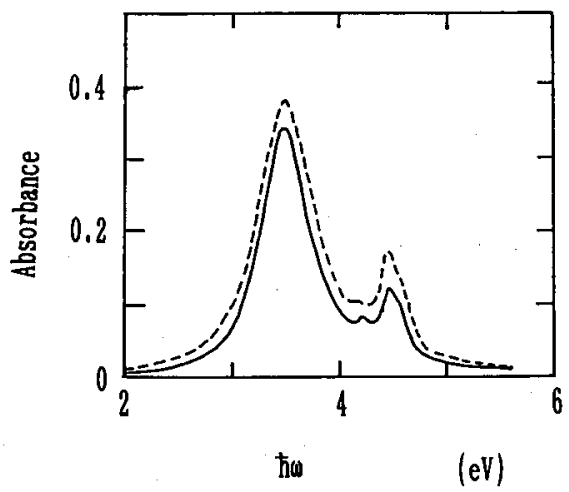

Fig. 5. Absorbance of normally incident light of angular frequency $\omega$ on an array of silver spheres of radius $S=80 \AA$ occupying randomly $75 \%$ of the sites of a square lattice with the lattice constant $a=200 \AA$ on a PVA substrate (full line). The broken line shows the absorbance by the layer of silver spheres alone.

broken line shows the absorbance of the array of metal particles on its own, the solid line shows the absorbance of the complete system (metal particles on the PVA slab) with multiple scattering of light between metal spheres and substrate taken fully into account. We see that at least in this case there is no significant effect to be had from the interaction with the substrate.

We have already mentioned that deviation from the spherical shape of the metallic particles could be important in the determination of the structure to be expected of the absorbance and reflectivity curves. The treatment of non-spherical particles can be done, at least for regular arrays of such particles, without much difficulty, at least in principle, provided one is prepared to allow for additional computing time. Such a calculation is presently in progress.

In the examples considered so far the transmitted and reflected light is carried by the specular $(g=0)$ beam. In our last example (Fig. 6) this is not the case. In this case light is incident normally (the electric field points along the $x$-direction) on a square array of spheres in the $x y$-plane. The spheres are made of a dielectric material with a refractive index $n_{M}=\sqrt{\varepsilon_{M}}=3.06$ which corresponds to the refractive index of $\mathrm{Al}_{2} \mathrm{O}_{3}$ in the microwave region. The surrounding medium has a refractive index $n=1.01$ which corresponds to thermal-compression-molded dielectric foam. The above values of the dielectric constants are those cited by Yablonovitch and Gmitter [8] in relation to their experiments on the photonic gap problem. We have taken $S / a=0.40$, where $S$ stands for the radius of the spheres and $a$ for the lattice constant. The reflectivity is plotted against $a / \lambda$, where $\lambda$ is the wavelength of the incident light, so that the results can be used in any other frequency region with appropriate scalling of $S$ and $a$. We note that when $a / \lambda<1$ the reflected (transmitted light) is carried by the specular $(g=0)$ beam but when 


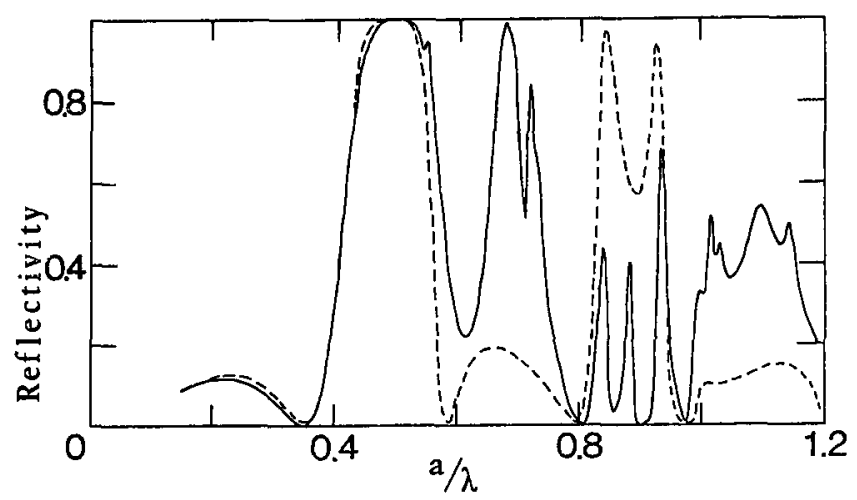

Fig. 6. Reflectivity of normally incident light of wavelength $\lambda$ from a square lattice of $\mathrm{Al}_{2} \mathrm{O}_{3}$ spheres in thermal-compression-molded dielectric foam. The ratio of the sphere radius $S$ to the lattice parameter $a$ is $S / a=0.40$.

$a / \lambda \geq 1$ this is shared between five $g$-vectors (those of lowest magnitude). The dotted curve is obtained in the dipole approximation $\left(\ell_{\max }=1\right)$, the solid line is obtained with $\ell_{\max }=4$ when convergence has been attained. The rich structure of the reflectivity curve is indeed remarkable. At this stage we are not able to present results for multilayers of such spheres, but work is now in progress in this direction and we hope to be able to do so in the very near future.

\section{References}

[1] A.J. Sievers, in Solar Energy Conversion, Ed. B.O. Seraphin, Springer, Berlin 1979.

[2] A. Modinos, Physica A 141, 575 (1987).

[3] N. Stefanou, A. Modinos, J. Phys., Condens. Matter (submitted for publication).

[4] W. Lamb, D.M. Wood, N.W. Ashcroft, Phys. Rev. B 21, 2248 (1980).

[5] N. Stefanou, A. Modinos, J. Phys., Condens. Matter (submitted for publication).

[6] J.B. Pendry, Low Energy Electron Diffraction, Academic Press, London 1974.

[7] A. Modinos, Field, Thermionic, and Secondary Electron Emission Spectroscopy, Plenum Press, New York 1984.

[8] E. Yablonovitch, T.J. Gmitter, Phys. Rev. Lett. 63, 1950 (1989).

[9] B.N. Persson, A. Liebsch, Phys. Rev. B 28, 4247 (1987).

[10] T. Yamaguchi, S. Yoshida, A. Kinbara, Thin Solid Films 21, 173 (1974).

[11] H.G. Craighead, G.A. Niklasson, Appl. Phys. Lett. 44, 1134 (1984); G.A. Niklasson, H.G. Craighhead, Thin Solid Films 125, 165 (1985). 